
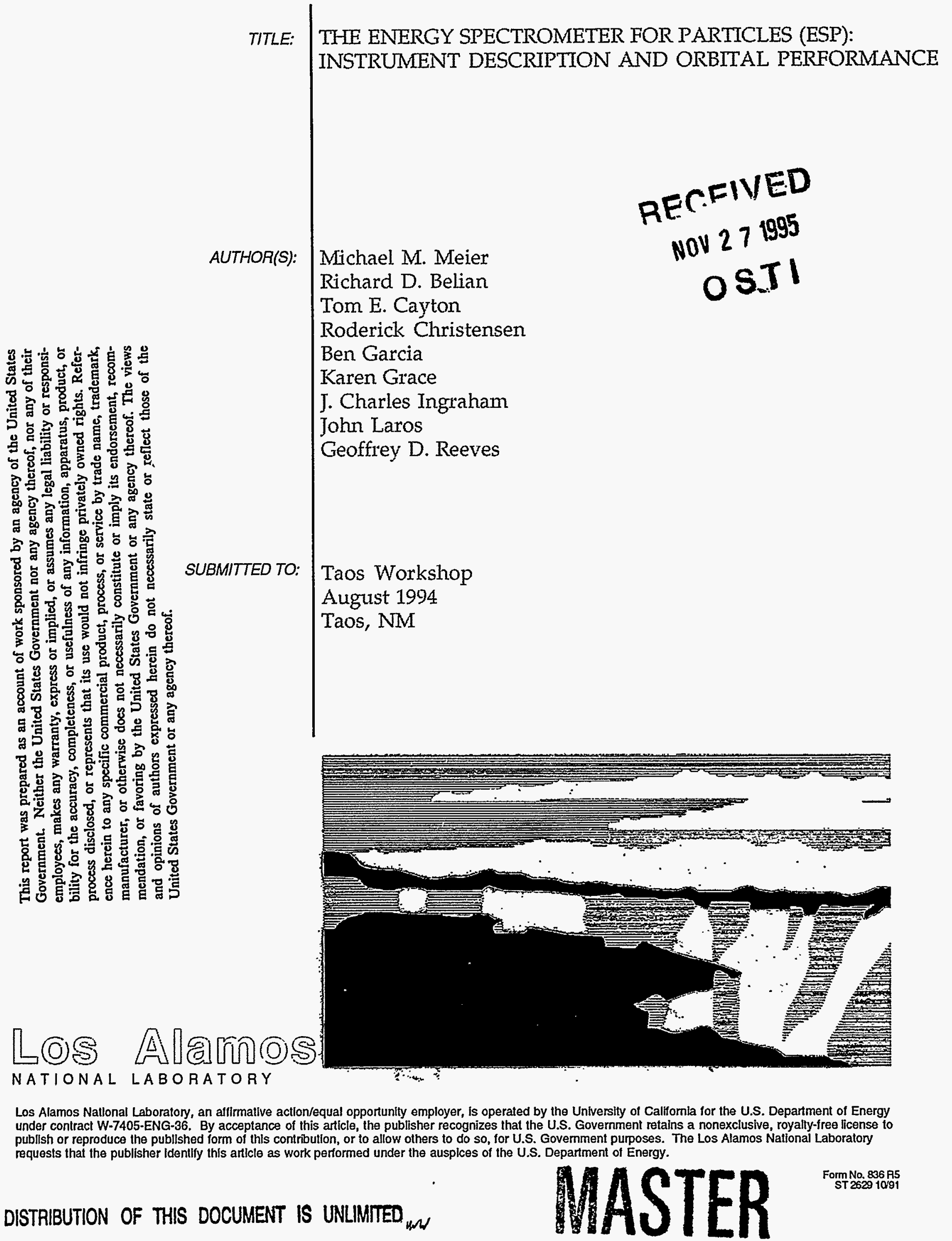

Los

Los Alamos Natlonal Laboratory, an aflirmalive action/equal opportunity employer, is operated by the University of California for the U.S. Department of Energy under contract W-7405-ENG-36. By acceptance of this article, the publisher recognizes that the U.S. Government retains a nonexclusive, royally-free license to publlsh or reproduce the publlshed form of this contribution, or to allow others to do so, for U.S. Government purposes. The Los Alamos National Laboratory requests that the publisher Identily this article as work performed under the auspices of the U.S. Department of Energy. 


\title{
The Energy Spectrometer for Particles (ESP): Instrument Description and Orbital Performance
}

\author{
MIKE MEIER, DiCK BELIAN, TOM CAYTON, ROD CHRISTENSEN, BEN GARCIA, KAREN \\ GRACE, CHUCK INGRAHAM, JOHN LAROS, AND GEOFF REEVES
}

Los Alamos National Laboratory, Los Alamos, New Mexico

\begin{abstract}
The ESP detector is functionally described, along with the pertinent orbital and spin properties of the spacecraft that supports it. The phoswiched plastic/BGO scintillators sensor design, electronic implementation, and resulting data types are recounted, and the ground calibration procedures are reported. Several illustrative examples of data are given, including the solar proton event of 29 September 1989, and the nearly periodic episodes of high relativistic electron flux that are associated with solar coronal holes which have been a dominant feature of the space weather over the past few years
\end{abstract}

\section{INTRODUCTION}

\section{Instrument Capabilities}

The ESP detector is an instrument capable of measuring the time-resolved energy spectra of electrons between 0.7 and $10 \mathrm{MeV}$ and of heavy charged particles. The energy range for protons is $11 \mathrm{MeV}$ to more than $100 \mathrm{MeV}$.

\section{Satellites and Orbital Parameters}

There are two instruments each on the satellites 1989-046, 1990-095, 1991-080, and 1994-085. They are mounted so the collimator axes are both in the plane perpendicular to the satellite spin axis, and separated by $180^{\circ}$. The satellite spin rate is approximately 10 seconds.

\section{Predecessors and Database}

The predecessors of the ESP detector, called SEE (Spectrometer for Energetic Electrons), have been flown on a number of geostationary satellites since mid-1979, including satellites 1979-053, 1981-025, 1982-019, 1984-037, 1984-129, and 1987-097. SEE data is still being returned from the latter two. The SEE detector was a simple modification of an existing instrument on the satellite, which modification provided a stacked pair of $1500 \mu \mathrm{m}(1000 \mu \mathrm{m}$ on later satellites) silicon solid-state sensors and collimator fronting an existing BGO crystal. The SEE detector provides energetic electron measurements from $\sim 1$ to $16 \mathrm{MeV}$ in 5 differential energy channels. With a minimal bit rate of 5 bps, it provides the 5 channels of data in 16 second accumulations. The SEE data have been produced continuously since mid1979 , initially by a single satellite, but by early 1982 by detectors aboard three satellites nearly equally spaced in geostationary orbit.

\section{Overall Design \\ INSTRUMENT}

The ESP detector is shown in cross section in Fig. 1. The penetrating charged particle sensor is a $3.81 \mathrm{~cm}$ diameter by $3.81 \mathrm{~cm}$ long bismuth germanate (BGO) crystal mechanically and optically coupled to a $0.63 \mathrm{~cm}$ thick plastic scintillator (BC400) which completely surrounds the BGO. A collimating aperture is coaxial with the scintillator axes and constrains charged particles in the energy range of interest to pass into the plastic scintillator and, if sufficiently energetic, into the BGO. The collimator

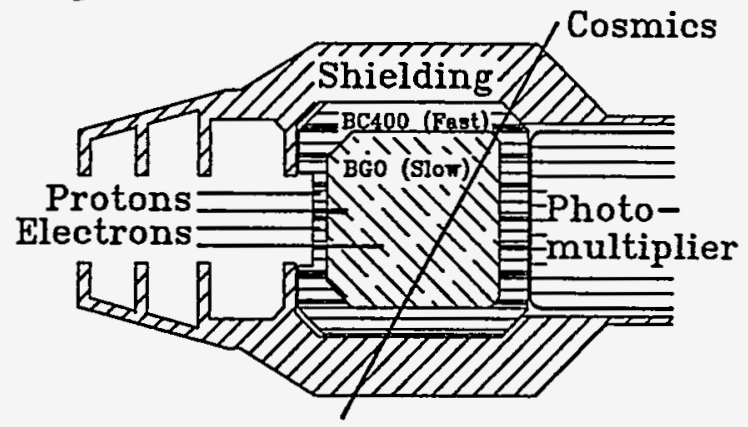

Figure 1. Cross section of the ESP phoswiched sensor, shielding, and collimation. 
provides a field of view with half angle of $11^{\circ}$. Galactic cosmic rays, with energies in the vicinity of $1 \mathrm{GeV}$, pass through the shielded assembly with only modest energy loss (less than $100 \mathrm{MeV}$ in the scintillator), and provide on-orbit gain calibration as discussed below. During periods when the count rates become too high to be reliable, the anode current in the photomultiplier that views the scintillators can be used as a measure of the dose rate in the detector.

\section{Sensor and Electronics}

Bismuth germanate $(B G O)$ is a dense material with a high stopping power for penetrating radiation and a moderate (approximately $300-$ ns) response time. The plastic scintillator (BC400), on the other hand, has low stopping power and a very fast (approximately 3-ns) response. The $\mathrm{BC} 400$ surrounds the $\mathrm{BGO}$ to provide charged-particle discrimination, achieved by "phoswiching" -. that is, an optical bonding of both scintillators to the same photomultiplier and separation of their responses by pulse-rise-time analysis. One-half inch of aluminum shielding, opaque to electrons with energies less than approximately $6 \mathrm{MeV}$ and protons of energy less than approximately $50 \mathrm{MeV}$, partially surrounds the assembly. The

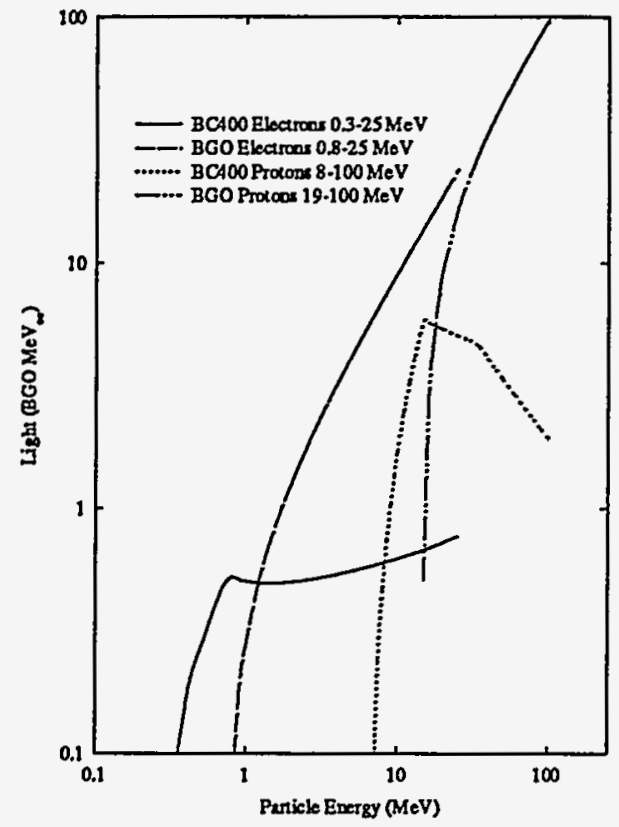

Figure 2. Light response of phoswiched sensors to electrons and protons. purposes of this shielding are to limit the total charged-particle flux incident on the sensor and to provide a directional charged-particle response. A cylindrical aperture in this shield, and a thinner $(0.32 \mathrm{~cm})$ area of the $B C 400$ immediately behind the aperture, act together as the charged-particle window through which pass most of the electrons and ions to be analyzed.

Figure 2 qualitatively illustrates the light production in each of the two phoswiched scintillators by the dominant radiation types. For collimated electrons with sufficient energy to penetrate the 0.020 -in. aluminum window, the $\mathrm{BC} 400$ light increases with energy to a maximum corresponding to the range energy of the plastic scintillator. As the energy continues to increase, the light decreases with $\mathrm{dE} / \mathrm{dx}$ for the plastic and then resumes a modest increase as bremsstrahlung becomes more important at higher energies. BGO light is produced for electrons with energies high enough to penetrate both the aluminum window and the $\mathrm{BC} 400$ layer and increases as the electrons range out in the BGO. At sufficiently high energy, the electrons are transmitted through the detector, and the light decreases with $\mathrm{dE} / \mathrm{dx}$ of the $\mathrm{BGO}$. At higher energies, comparable behavior is observed for heavier charged particles because their ionization losses are higher than those for electrons.

Figure 3 shows the relationship of the $\mathrm{BC} 400$ and BGO light for the two particle species. The individual species can clearly be identified by their unique dependencies on BC400 and BGO light. In particular, BC400 light levels will distinguish protons that generate more than about $0.7 \mathrm{MeV}_{\mathrm{ee}}$ * in the $\mathrm{BC} 400$ from electrons over a broad energy range, except at the highest energies where the protons approach minimum ionization.

After the light is detected and converted to an amplified current pulse in a photomultiplier, the pulses are amplified in both a broadband (all frequencies) and a fast (low frequency rejection) amplifier that have as their respective inputs the anode and tenth (last) dynode signals. The amplitude of the broadband amplifier output, B, is proportional to the total signal, that is, the sum of the fast plastic response and slow

$*_{1} \mathrm{MeV}_{\mathrm{ee}}$ is the amount of light produced by a $1-\mathrm{MeV}$ electron that stops in the BGO. 


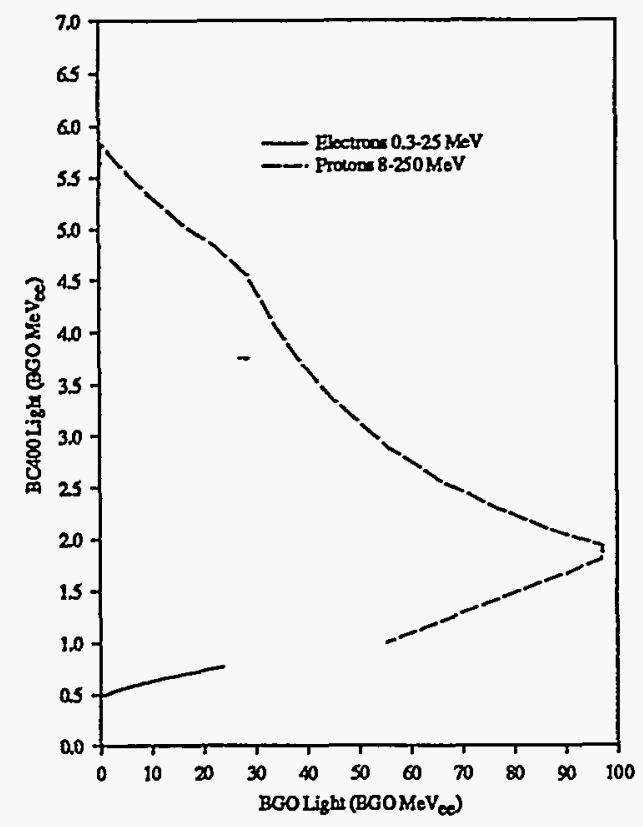

Figure 3. Relative "fast" and "slow" light responses for protons and electrons in the sensor. A threshold corresponding to about 1 $\mathrm{MeV}_{\mathrm{ee}} \mathrm{BC} 400$ permits particle discrimination.

response of the BGO:

$$
B=G_{B}(B G O+\alpha P) ; \alpha=1,
$$

where $G_{B}$ is the combined gain of the anode and broadband amplifier, $\mathrm{P}$ is the fast component of the plastic scintillator response, BGO is the slow response of the $B G O$, and $\alpha$ is the factor to account for the small broadband gain difference between fast and slow components.

The output of the fast amplifier, $\mathrm{F}$, is

$$
F=G_{F}(P+\varepsilon B G O) ; \varepsilon \ll 1,
$$

where $G_{F}$ is the combined gain of the dynode and fast amplifier and $\varepsilon$ is the small amount of BGO component that is transmitted through the fast amplifier and is of the order $\mathrm{T}_{\mathrm{F}} / \mathrm{T}_{\mathrm{BGO}}$. The time constant of the fast amplifier, $\mathrm{T}_{\mathrm{F}}$, is approximately $10 \mathrm{~ns}$, and the decay time of light from $\mathrm{BGO}, \mathrm{T}_{\mathrm{BGO}}$, is approximately $300 \mathrm{~ns}$.

$B$ and $F$ for electrons and protons have a qualitatively similar relationship to that seen in Figure 3, except for the mixing introduced by the electronics. Although integral pulse-height discrimination can be accomplished over reasonable energy ranges with these outputs, there are other considerations. Uncollimated very high energy protons that penetrate the shield can have long path lengths in the $\mathrm{BC} 400$ and thus generate intense light signals. These distort the proton pulse-height spectrum, making it more difficult to use as a calibration reference. Electrons that excessively straggle in the $\mathrm{BC} 400$ can mimic proton events and further confuse the spectrum.

Generating a third pulse, $S$, that is the difference between an appropriate fraction $f$ of the $F$ pulse and the $B$ pulse, can eliminate both problems:

$$
\begin{gathered}
S=B-f F \\
=B G O\left(G_{B}-f \varepsilon G_{F}\right)+P\left(\alpha G_{B}-f G_{F}\right),
\end{gathered}
$$

where $f$ is adjusted to eliminate the coefficient of $\mathrm{P}$ in $\mathrm{S}$,

$$
f=\alpha \mathrm{G}_{\mathrm{B}} / \mathrm{G}_{\mathrm{F}}
$$

and $S$ becomes a measure of the BGO light, uncontaminated by any plastic contribution:

$$
S=B G O(1-\alpha \varepsilon) G_{B} \text {. }
$$

Thus the pulse-height spectrum of $S$ is free of the distorting effects of the potentially large light contributions from the plastic scintillator. Additionally, use of the subtracted pulse reduces the dynamic range requirements of the following amplifier. Figure 4 shows the relation of $S$ and F. Finally, the discrimination scheme is somewhat improved. Because $S$ has no fast component, cleaner discrimination can be achieved at low pulse heights.

\section{Data Types}

Using these pulses, we can identify particle species over broad energy ranges. Two discriminators are set for each of the circuits that process $F$ and $S$ : the lower level discriminator for the fast pulse (FLLD) is set to $0.3 \mathrm{MeV}_{\mathrm{ee}}$, and the upper level discriminator for the fast pulse (FULD) is set to $1 \mathrm{MeV}_{\mathrm{ee}}$; the lower level discriminator for the slow (subtracted) pulse (SLLD) is set to $1 \mathrm{MeV}_{\mathrm{ee}}$, and the upper level discriminator for the slow pulse (SULD) is set to $10 \mathrm{MeV}_{\text {ee. }}$. The event parameter space defined by these discrimination level is schematically represented in Figure 4, along with the particle nomenclature scheme. 


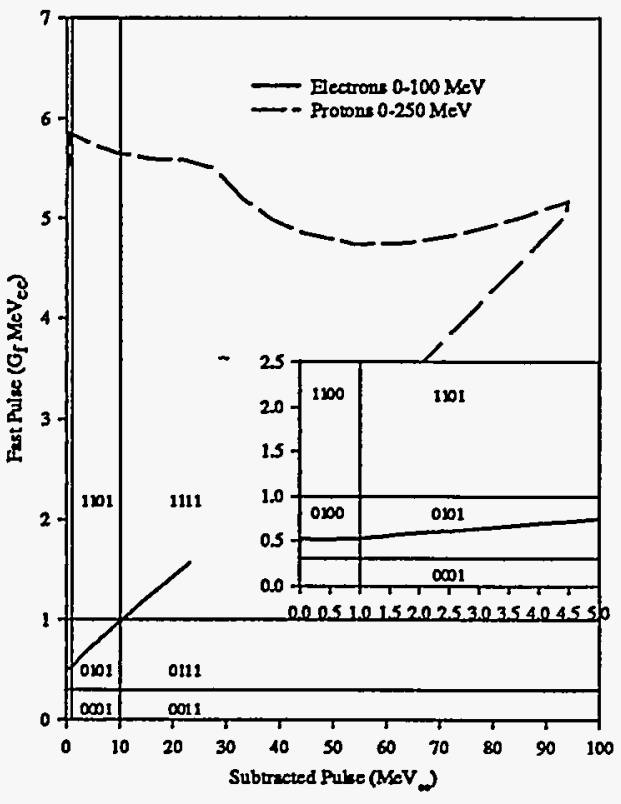

Figure 4. Electronics processed "fast" and "subtracted" pulses showing the thresholds and related regions into which the data are binned.

Events with fast light between 0.3 and 1.0 $\mathrm{MeV}_{\mathrm{ee}}$ are identified with electrons, and lie in the lower horizontal band; and events with fast light above $1.0 \mathrm{MeV}_{\mathrm{ee}}$ are identified with protons and lie in the upper horizontal band. The BGO is sensitive to the bremsstrahlung produced by relativistic electrons that bombard the spacecraft, and these $\gamma$-rays are rejected by their lack of significant BC400 amplitude. An event is labeled by the combination of level discriminators that it triggers, "FfSs," where $\mathrm{F}=$ $1 / 0$ corresponds to FULD triggered/nottriggered, $\mathrm{f}=1 / 0$ to FLLD triggered/nottriggered, $S=1 / 0$ to $S U L D$ triggered/nottriggered, and $s=1 / 0$ to SLLD triggered/nottriggered. Each allowed combination corresponds to one of the six areas of Fig. 4 that are defined by these four level discriminators. The figure shows that 0100 corresponds to electrons that have deposited less than $1 \mathrm{MeV}_{e e}$ of light in the BGO, 0101 to electrons that have deposited 1-10 $\mathrm{MeV}_{\mathrm{ee}}$ of light in the BGO, and so on.

For level discriminator (LD) combinations corresponding to $1.8 \mathrm{MeV}$ to $10 \mathrm{MeV}$ electrons (0101) and to $20 \mathrm{MeV}$ to $>100 \mathrm{MeV}$ protons (1111), an analog-to-digital converter (ADC) measures the energy spectra, and provides a continuous in-flight calibration of the instrument gain. Table 1 lists the possible LD combinations, the dominant radiation type causing each combination, and the processing of the ensuing signals. For example, primary cosmic rays (mostly extremely high energy protons) pass completely through the sensor, depositing only a small fraction of their energy along their paths. Because they will almost always traverse two layers of $\mathrm{BC} 400$, they will produce pulses having a large fast component (1100, 1101, or 1111). Lower-energy protons and other ions (10-50 MeV) can enter only through the aperture and will stop in the thin section of $\mathrm{BC} 400$ or in the BGO. However, because they are more heavily ionizing (that is, they deposit more energy per unit path length), they will also produce pulses with a large fast component. Thus, the ion channels will count an essentially constant background of primary cosmic rays plus a more variable lower-energy ion component. Electrons entering the aperture with energy sufficient to trigger the FLLD (approximately $0.5 \mathrm{MeV}$ ) are not heavily ionizing and will produce only a small fast component. Accordingly, the electron signature is 0100,0101 , or 0111 , depending on the electron energy. A summary of the data types (telemetry channels), the particles and energies that dominate them, and their respective accumulation times are listed in Table I. Of course, this particle discrimination scheme is not perfect. Electrons can be appreciably deflected as they traverse the BC400. Because deflection lengthens the effective paths of electrons, their energy deposition will be greater and they will be mistaken for protons. Additionally, various physical processes will unavoidably cause coupling between supposedly independent data channels. For example, sufficiently high energy protons can produce nearly any secondary radiation by nuclear processes. The magnitudes of such effects are determined by ground testing and computer simulations.

\section{Calibrations}

The electron and proton responses of the ESP channels have been estimated using Monte Carlo techniques and compared to the experimentally measured quantities. The electron responses were measured using the Aerospace Inc. monoenergetic electron source for electron energies up to $3.2 \mathrm{MeV}$. The results 
are shown in Figure 5. The electron energy was varied, and the fraction of incident electrons counted in each channel was recorded. These data are plotted in the figure along with the Monte Carlo estimates. The latter give a good representation to the data and are used in the conversion of count rate to flux. As mentioned previously, multiple scattering in the BC400 can falsely identify electrons as protons, because the light from such _events can trigger the Fast Upper Level Threshold. Such events are also successfully represented by the calculations, as shown in Figure 6.
The proton response is measured with very low intensity ( $<100$ protons/second) beams at a tandem Van de Graaff accelerator. Beams of protons with energies as high as $22 \mathrm{MeV}$ are produced there, and the thresholds for 1100 , 1101 , and 1111 channels can thus be measured.

The dose response of the detectors are calibrated at the Los Alamos HS-4 Radiation Instrument Calibration facility. ${ }^{60} \mathrm{Co}$ sources are used to calibrate the anode current response for dose rates between $10 \mathrm{mR} / \mathrm{hr}$ and $400 \mathrm{mR} / \mathrm{hr}$.

TABLE I. Radiation Measurement Capability of ESP

\begin{tabular}{|c|c|c|c|l|l|}
\hline FULD (F) & FLLD (f) & SULD (S) & SLLD (s) & \multicolumn{1}{|c|}{ Dominant Radiation Type } & \multicolumn{1}{|c|}{ Processing } \\
\hline 0 & 1 & 0 & 0 & $0.7-1.8-\mathrm{MeV}$ electron & To scaler (8.192-s accum.) \\
\hline 0 & 1 & 0 & 1 & $1.8-11-\mathrm{MeV}$ electron & $\begin{array}{l}\text { To scaler (8.192-s accum.) and } \\
\text { ADC (channels E 2-8) }\end{array}$ \\
\hline 0 & 1 & 1 & 1 & $11-25-\mathrm{MeV}$ electron & To scaler (8.192-s accum.) \\
\hline 1 & 1 & 0 & 0 & $\begin{array}{l}11-15-\mathrm{MeV} \text { proton + cosmic- } \\
\text { ray primaries }\end{array}$ & To scaler (8.192-s accum.) \\
\hline 1 & 1 & 1 & 1 & $\begin{array}{l}\text { >19-MeV proton + cosmic- } \\
\text { ray primaries }\end{array}$ & $\begin{array}{l}\text { To scaler (8.192-s accum.) and } \\
\text { ADC (channels PHA 8-15) }\end{array}$ \\
\hline $\mathrm{X}^{\mathrm{a}}$ & 1 & $\mathrm{X}$ & $\mathrm{X}$ & Any charged particle & $\begin{array}{l}\text { To scaler (0.256-s accum.) for } \\
\text { FLLD channel }\end{array}$ \\
\hline & Any LD & & & Any particle & To scaler (8.192-s accum.) \\
\hline
\end{tabular}

a)

$\mathrm{X}$ can be either 0 or 1

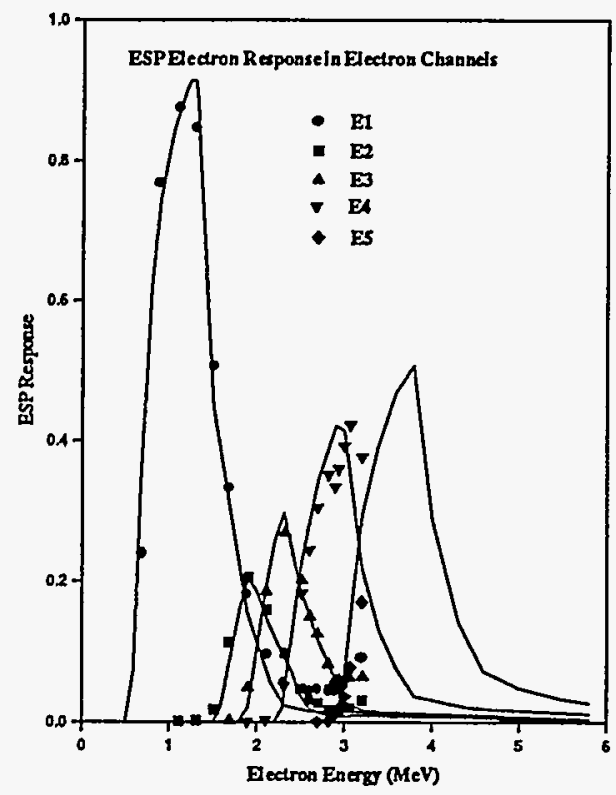

Figure 5. Measured and calculated electron responses of the ESP electron channels.

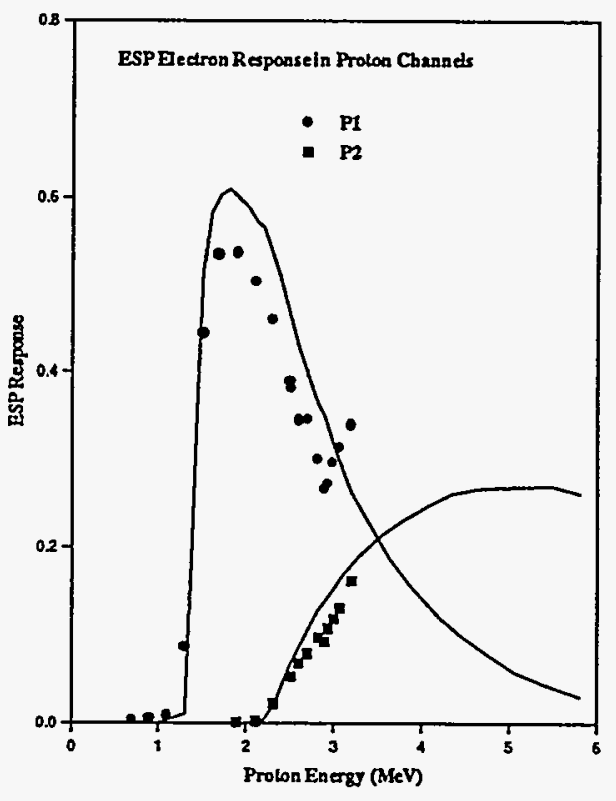

Figure 6. Measured and calculated electron responses of the ESP proton channels. 
The responses to dose, along with the fitted calibration curves are shown in Figure 7.

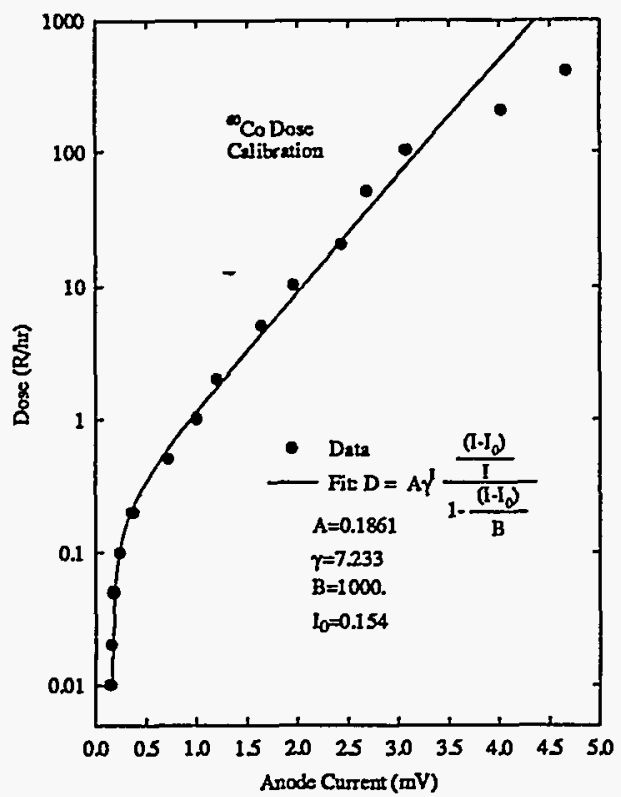

Figure 7. ESP Dose-anode current relation. The fit to the data provides a conversion up to doses of $100 \mathrm{R} / \mathrm{hr}$.

There are three complementary procedures for in-flight calibration. The first involves monitoring the pulse amplitude distribution caused by primary cosmic rays, which can be done whenever the flux of lower-energy ions is small, i.e. in the absence of solar energetic particle events. This method relies on the fact that the actual energy distribution depends only on the detector geometry and has a pronounced peak at $40 \mathrm{MeV}$ for this instrument. The second calibration procedure (CAL 04) involves injecting, by command, a series of pulses into the fast and slow preamplifier inputs in order to verify that the electronics are operating correctly. The pulses are charge divided and ramped in amplitude in such a way that most of the detector channels are stimulated with a known number of counts. Finally, an LED visible to the photocathode is pulsed on for several seconds to test the response of the anode current to high light levels.

\section{ILLUSTRATIVE DATA}

\section{Solar Energetic Particle Events}

The first ESP detector was turned on in late September 1989, just in time to record the onset of a very large solar-energetic-particle (SEP) event that began on 29 September [J. R. Asbridge]. The early phase of this event is illustrated in Figure $8 \mathrm{a}$ where the count rate of the summed proton channel (Ep $>19 \mathrm{MeV}$ ) is plotted as a function of time for the 24 hours beginning 00:00:00 UT on that day. The usual count rate for this channel is relatively constant at about $40 \mathrm{c} / \mathrm{s}$, and is due to very high energy cosmic rays which deposit only a small fraction of their energy in the BGO and plastic
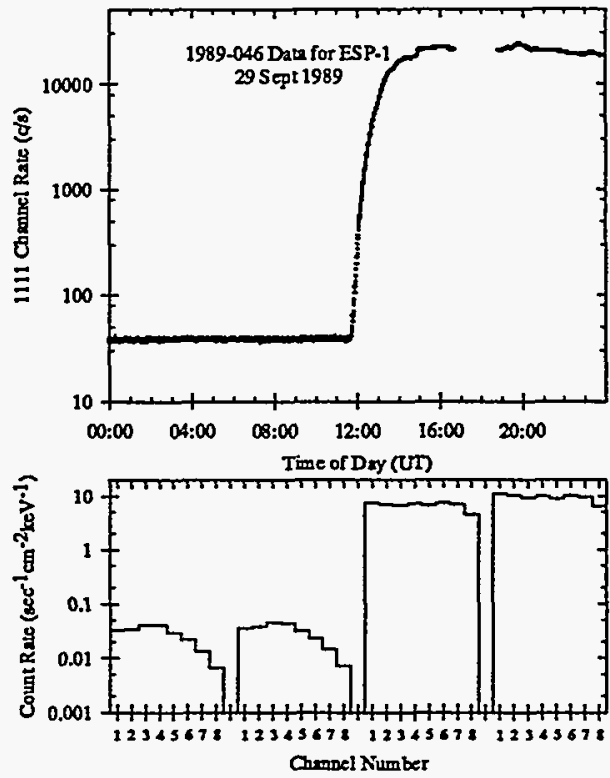

Figure 8. a) The count rate in one of the ESP "1111" channels on 29 September 1989. b) Six hour average pulse height spectra for protons between 17 and $65 \mathrm{MeV}$.

scintillators. The SEP event evidently began about mid-day with a very fast rise of the solar protons (probably generated by the solar flare at around 12:00), followed by a second surge of particles at 13:00. The very high count rate that would have resulted from the latter is suppressed somewhat due to saturation effects in the sensor and associated electronics. The 13:00 rise in flux is thought to be due to protons that are accelerated by processes in the high speed solar wind stream produced by an associated coronal mass ejection. Figure $8 \mathrm{~b}$ shows the measured six-hour average proton energy spectra for the four quarters of the day. The first two spectra were taken just prior to the onset of the SEP event, and largely reflect the distribution of energies lost by minimum ionizing particles as 
they traverse the possible path lengths through the detector. The other two were taken after the solar particles had reached geosynchronous orbit. In this case, the spectra are dominated by energetic solar protons that enter the detector through the collimator. The lower bin bounds for channels PHAl-8 are $19,30,36,40,45,49$, 54 , and $62 \mathrm{MeV}$.

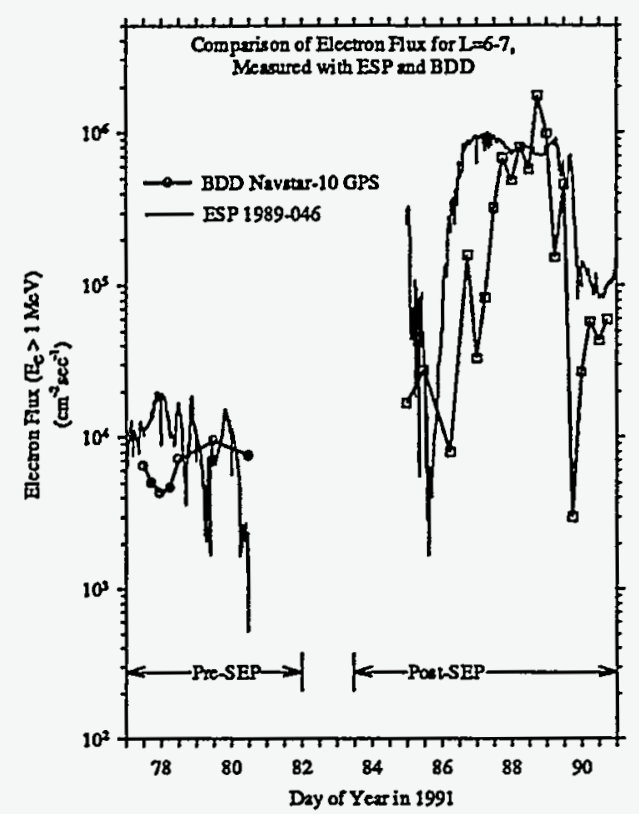

Figure 9. Comparison of ESP and BDD electron data for the SEP event of 24 March 1991.

\section{March 1991}

The March 24, 1991, Giant Magnetic Storm and accompanying Solar Energetic Particle Event (SEP), provide data over a wide dynamic range of energy and count rate that can be used to compare absolute ESP electron flux measurements with those made by other instruments on other satellites, for the purpose of verifying calibrations. Figure 9 shows such a comparison of the ESP-determined omnidirectional electron flux $(E>1 \mathrm{MeV})$ with that determined from another instrument, BDD, on the Navstar 10 Global Positioning Satellite (see [J. C. Ingraham, et al.] in this proceedings for further description of the BDD measurements). The plotted BDD data points are selected only for those times when the satellite is near to the $L \sim 6.5$ charged particle drift surface, which also passes through the geosynchronous satellite orbit of ESP. Prior to, and after the SEP the agreement between the two fluxes is quite good even though the magnetic latitudes of the two satellites at the times of comparison are very different (BDD: $38^{\circ}$; ESP: $\sim 0^{\circ}$ ). The delay of the flux increase at BDD around day 87 relative to the flux increase at ESP around day 86 appears to be real and is discussed in more detail, elsewhere [J. C. Ingraham, et al.]. However, the apparent close agreement between ESP and BDD for days 8889 is an artifact due to depression of the ESP

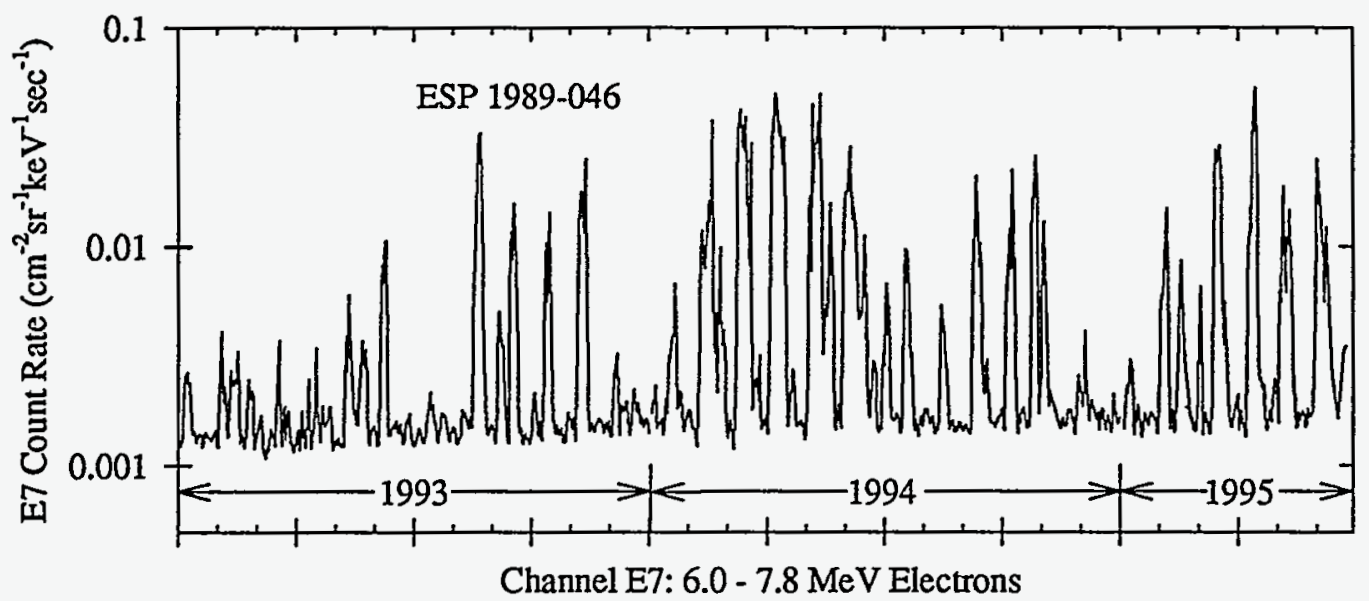

Figure 10. Relativistic electron flux history for 1993 to present. The nearly periodic structures are correlated with the presence of solar coronal holes. 
output count rate at these very high input count rates. A BDD flux (at the higher magnetic latitude) that is lower than the ESP flux, as is the case here, is consistent with the measured pitch angle distribution at the geomagnetic equator for this period [J. C. Ingraham, et al.] that favors the pitch angles perpendicular to the Earth's magnetic field.

\section{January - Present: Solar Coronal Holes and} Trapped Relativistic Electrons

It is well known that sustained, high fluxes of relativistic electrons produced in conjunction with high-speed streams in the solar wind tend to occur in the declining phase of the sunspot cycle. Such a period is occurring now. In figure 10 is shown the time history of $6-7.8 \mathrm{MeV}$ electron flux for 1993, 1994, and about half of 1995. One can see the periodic increases (period of roughly 27 days) in flux that occur throughout 1993, and which tend to maximize in early 1994. These are the highly penetrating electrons that are thought to be responsible for many spacecraft anomalies and even failures that occurred during early 1994 . Although the maximum flux values were again quite high in early 1995 , one can see that the high fluxes are not as sustained as the early 1994 episode. Of course, the periodicity comes about because the electrons are, in a yet to be explained manner, generated in the magnetosphere during the periods of high solar-wind speed. These high speed solar winds are generated by solar coronal holes that rotate with the sun. A single active area on the sun may be responsible for a nearly periodic series of relativistic electron events as the hole rotates through the earth's magnetic longitude with the sun's 27 day period.

\section{CONCLUSIONS}

The design and operation of the ESP detectors has been described along with the experimental calibrations that confirm the calculated response. Several examples of orbital operation during times of interest in the past few years have been used to illustrate capabilities and limitations.

\section{REFERENCES}

Asbridge, J. R., Bame, S. J., Belian, R. D., Drake, D. M., Guyker, F., Laros, J. G., The Solar Energetic Particle Events of October 1989: Observations by Los Alamos Instrumentation, Los Alamos National Laboratory Unclassified Report LA-UR 89-3864, 1989.

Ingraham, J. C., Cayton, T. E., Belian, R. D., Christensen, R. A., Guyker, F., Meier, M. M., Reeves, G. D., Brautigam, D. H., Gussenhoven, M. S., and Robinson, R. M., Multi-Satellite Characterization of the Large Energetic Electron Flux Increase at $\mathrm{L}=4-7$, in the Five-Day Period Following the March 24, 1991, Solar Energetic Particle Event in Proceedings of the Taos Conference, August 14-19, 1994 edited by G. D. Reeves 